\title{
NOUVELLES DIVERSES
}

\author{
INFLUENCE DE CERTAINS FACTEURS \\ "NON SCIENTIFIQUES " \\ SUR LA PRATIQUE ET LES NORMES \\ DE RADIOPROTECTION
}

Extraits de la conférence prononcée par Lauriston S. TAYLOR

au Congrès de l'I.R.P.A. à Jérusalem le 10 mars 1980

\section{NOTE DE LA RÉDACTION}

A l'ouverture du $5^{\mathrm{e}}$ Congrès de l'Association internationale de Radioprotection (I.R.P.A.) tenu à Jérusalem en mars 1980, les congressistes ont eu le privilège d'entendre une conférence exceptionnelle de Lauriston TAYLOR, ancien président du « National council on radiation protection and measurements (N.C.R.P.). Lauriston TAYLOR peut être considéré comme l'un des plus grands spécialistes des problèmes de la protection contre les rayonnements ionisants.

Le texte intégral de cette conférence a été publié dans les actes du congrès ainsi que dans la revue Health Physics (vol. 39, décembre 1980, p. 851). Le reproduire à nouveau dans nos colonnes pourrait paraître fastidieux, notamment pour des personnes à la recherche d'une synthèse des problèmes majeurs posés par la radioprotection. Aussi avons-nous choisi de résumer ce texte pourtant passionnant d'un bout à l'autre, tant sur le fond que pour la grande qualité de l'écriture. Les passages les plus importants sont reproduits in extenso, les autres étant condensés pour conserver une certaine unité à l'ensemble.

Le lecteur pourra constater que les problèmes techniques abordés par Lauriston TAYLOR sont peu nombreux; par contre, l'auteur expose avec gravité le rôle joué dans l'information du public par la presse et certains spécialistes isolés, ainsi que le poids des lois et des règlements dans un domaine pourtant remarquablement exploré par les responsables.

Face à l'inquiétude du public devant les rayonnements, l'auteur recommande aux scientifiques de poursuivre leur effort d'information, avec conscience et dignité, et si possible avec les media, les uns et les autres ne pouvant s'ignorer.

\section{INTRODUCTION}

"Nous savons aujourd'hui tout ce qui est nécessaire pour assurer une protection adéquate contre les rayonnements ionisants. Je pose donc la question : pourquoi $y$-a-t-il un problème des rayonnements et où se situe-t-il? "

Il faut dépasser le domaine scientifique pour embrasser les explications philosophiques, sociologiques, politiques, économiques et morales du problème des rayonnements. Vis-à-vis du public, l'information et l'élaboration de mesures 
d'intérêt général doivent être la règle commune pour les rayonnements mais aussi pour toutes les substances toxiques.

L'essentiel, pour les spécialistes de la radioprotection est de savoir se garder de toute attitude de condescendance et de tout ce qui ressemblerait à une promotion personnelle.

\section{LE PROBLÈME SCIENTIFIQUE DES SEUILS}

Nous disposons d'une masse considérable de faits et de connaissances concernant les effets ionisants chez l'homme et chez l'animal, bien supérieure à ce que l'on sait sur les agents toxiques en général. Le public, pourtant, présente une attitude irrationnelle dans sa soif de savoir, et a le sentiment d'être trahi ou abusé.

"Un bon exemple nous en est donné actuellement par la controverse sur les effets des rayonnements délivrés à faibles doses. Si ce n'étaient quelques commissions parlementaires plus intéressées par les gros titres que par les faits, aidées et encouragées par une presse complaisante et par des " scientifıques " en mal de publicité, il est probable que les travaux sur la question iraient leur rythme, à la mesure de leur importance, dans le ronronnement des commissions et réunions scientifıques normales. Ce n'est pas qu'elle soit sans importance mais, dans l'ordre des priorités, elle ne saurait avoir le pas sur tant d'autres accidents auxquels est exposé l'homme. Les effets des rayonnements sont généralement proportionnés à la dose lorsqu'ils ont été délivrés à un débit élevé en quantités modérées, c'est-àdire inférieures à $100 \mathrm{rads}$, dans les régions observées. Une proportionnalité est diffıcile à établir dans la mesure où les rayonnements ne produisent pas nécessairement les effets habituellement constatés sur la partie du corps exposée, mais peuvent affecter toute autre région. Nous postulons cependant pour les besoins de la protection que, pour une intensité de dose élevée, l'effet sera proportionnel à la dose." "

Un temps de latence sépare le moment de l'exposition et la manifestation des effets; réduit à quelques minutes ou à quelques heures pour les doses très fortes (supérieures à $500 \mathrm{rads}$ ), il peut être de plusieurs décennies pour les doses inférieures à 50 rads, voire encore supérieur pour des doses plus faibles. Compte tenu d'autres causes produisant les mêmes effets que les rayonnements, il convient d'examiner de grandes populations d'individus pour établir, de manière statistique, une relation significative entre les doses et les effets. Pour une exposition de quelques rads, ce nombre peut atteindre plusieurs millions de personnes.

L'existence d'un seuil au-dessous duquel aucun effet n'apparait a été établie pour certains effets dans le domaine génétique et pour diverses affections cutanées. Dans le cas général, il peut exister un seuil à un niveau trop bas pour que nous puissions l'observer. On est alors amené à poser en postulat qu'il n'existe pas de seuil et que toute dose peut produire un effet, même indécelable. Mais alors, "dans quelle mesure peut-on réduire les risques d'un certain taux 
lorsque, dans la région considérée, ou identiflée, aucun effet n'a été observé? La question est dès lors : à quoi correspond la moitié de ce qui ne peut être mesuré

"Les effets des doses ne sont pas cumulatifs. Il doit exister des processus de réparation, de restauration ou de remplacement des cellules tant génétiques que somatiques, ne serait-ce que pour les raisons qui fondent les techniques modernes de radiothérapie. On sait depuis au moins 50 ans que, dans le cas de la radiothérapie appliquée à une tumeur et aux tissus périphériques, la quantité totale des rayonnements peut être considérablement accrue grâce à la simple introduction de

- " pauses " entre chaque traitement. Sans processus de restauration cellulaire, il n'y aurait pas raisonnablement de radiothérapie possible, ce qui n'est assurément pas le cas.

" Les relations dose-effet nous sont aujourd'hui suffisamment bien connues pour que nous puissions affurmer sans équivoque qu'au moins pour les rayonnements à faible TLE, les relations ne sont pas strictement linéaires sur l'essentiel de la trajectoire et que, même pour les doses élevées, elles ne sont probablement pas linéaires. En général, La déviation par rapport à la linéarité a été telle que les normes de protection sont apparues plus prudentes ou plus restrictives que ne le laissait entrevoir la simple relation linéaire. "

Cette hypothèse de la relation linéaire, prise à la lettre, conduit à des restrictions inutiles et sans fondement de l'utilisation des rayonnements ionisants. Nous admettons que les risques sont décelables et identifiables au-delà de 200 rads délivrés à débit élevé. Par contre, nous ne sommes pas en mesure de déceler ou d'identifier les effets au-dessous de 25 rads délivrés à faible débit. D'autre part, nous n'envisageons que les effets nocifs ou considérés comme tels en supposant qu'ils le sont tous nécessairement.

\section{ASPECTS NON SCIENTIFIQUES DE LA RADIOPROTECTION}

On s'est rapidement rendu compte que les données et le raisonnement scientifıque n'étaient pas seuls à conduire à l'établissement des normes. "Dès 1957, je déclarais : "la radioprotection n'est pas seulement affaire de science, c'est une question de philosophie, de morale et d'information au plus haut niveau." J'avais ajouté, par la suite, "d'économie, de politique et d'action publique ", mais ce sont tous des éléments d'une approche idéologique. "

L'hypothèse d'absence de seuil conduit à supprimer la démarcation entre les zones où les effets sont scientifiquement prouvés, et les autres. On décide alors que les effets sont présumés exister. Un tel postulat peut émouvoir le scientifique, tout comme le profane qui lui fait confrance.

De plus, l'évaluation d'un risque généralisé à la population du globe donne un chiffre terriblement élevé et inquiétant.

L'établissement des normes à partir des hypothèses sur les dommages et les effets passe par l'acceptation du risque, donc par une analyse entre le bénéfice de l'action et ses conséquences. Mais le public ne sait pas apprécier ses actions en termes de risques. En tout cas, il s'en garde bien lorsqu'il s'agit pour lui de skier, de faire de l'alpinisme ou de fumer. Or, il est bien connu que ce sont là des activités à haut risque. 
"L Lutilisation médicale est la seule application où les risques, si risques ii y a, sont compensés par le profıt que la personne en situation de risque peut en retirer. C'est également dans le domaine médical que l'abstention - renoncer, par exemple, à un examen radiologique - peut comporter plus de risques pour l'individu que les rayons eux-mêmes. "

Pour compenser cette argumentation scientifique insuffisante, les scientifiques ont été contraints de fournir au public ou au patient une information couvrant le maximum de renseignements, le premier d'entre eux étant certainement qu'aucun dommage n'a encore été identifié comme imputable aux rayonnements parmi le personnel travaillant dans le cadre des normes fixées par la Commission internationale de protection radiologique (C.I.P.R.) dès 1934. En 40 ans de recherches, les théories concernant les personnes victimes de radiolésions doivent être considérées comme le fruit pur et simple de l'imagination.

"Une diffıculté peut naître ici de l'attitude actuelle du public qui soupçonne toute personne travaillant dans un domaine particulier (exemple : rayonnements) d'être impliquée dans un conflit d'intérêts dès lors qu'elle est associée au processus de décision. En fait, compte tenu de leur information de base et de leur sens fondamental de l'objectif, on pourrait faire valoir à juste titre que les scientiflques en tant que tels sont aussi dépourvus d'intérêts spéciaux que n'importe quel autre groupe.

"En dehors des scientifıques expérimentés, formés à la radioprotection, où puiser notre information? Personnellement, je suis persuadé que nous devrions nous tourner vers le grand public en général qui, dans sa majeure partie, peut être tenu pour bien intentionné et sincère encore que rarement bien informé des problèmes de rayonnements qu'il a à considérer. Qu'il prenne la forme de démarches individuelles ou collectives, le concours du public peut s'avérer précieux en matière de radioprotection s'il est convenablement guidé dans le domaine technique à l'abri des émotions, des déviations illogiques et inacceptables et de toute promotion personnelle. J'insiste sur l'utilisation du public dans l'élaboration de notre philosophie globale de la radioprotection. C'est une situation qui appelle une nouvelle approche de notre processus d'éducation du public et elle couvre de nombreux domaines d'intérêt autres que les rayonnements ionisants. Cependant, pour ce qui concerne les rayonnements, c'est un domaine où les responsables de la radioprotection doivent trouver leur place et y travailler avec autant de prudence et d'application qu'ils n'en ont mis à les contrôler, les mesurer et les analyser.

"Pour revenir à la question philosophique fondamentale de l'établissement de normes de protection, nous pouvons, en simplifiant largement, ramener le problème à un choix entre deux options. L'une consiste à faire plus ou moins sienne la théorie actuelle selon laquelle la relation dose-effet serait exclusivement linéaire et sans seuil. Cependant, ce faisant, nous devons prendre à l'avenir des mesures plus spécifıques pour attirer l'attention du public sur le fait : $1^{\circ}$ que cette théorie reste à démontrer; $2^{\circ}$ qu'elle n'est utilisée que parce que nous ignorons la relation précise; et $3^{\circ}$ qu'elle est probablement prudente pour la plupart des besoins pratiques. Nous devons en même temps nous arréter, et arrêter les autres, de livrer au grana public des estimations trop précises concernant le nombre probable de personnes 
succombant à une dose de $1 \mathrm{mrem}$ à la suite d'une fuite de réacteur ou à une dose de 5 mrem délivrée sans nécessité à un patient en plus d'une dose inévitable de 50 mrem par exemple. Des millions de passagers aériens sont, dans les avions volant à haute altitude, soumis à des doses comparables et les équipages à des doses bien plus élevées; on s'est jusqu'à présent peu préoccupé de cette situation. Il n'est toutefois pas dans notre intention de prendre argument de ce qui précède pour plaider en faveur d'un assouplissement des normes ou d'une tolérance des négligences dans l'utilisation des rayonnements. C'est seulement un exemple de la diversité des approches philosophiques touchant les risques, réels ou imaginaires, inhérents aux rayonnements.

" D'autre part, nous devons trouver des moyens acceptables pour mettre un terme ou réagir aux bavardages interminables de quelques-uns, quels que soient les motifs qui peuvent les porter à exciter, troubler, couper le public des techniciens eux-mêmes qui sont les mieux à même de l'informer correctement et de l'instruire.

"Une diffıculté fondamentale consiste à distinguer entre les bavardages pernicieux d'une part et une idée à l'occasion utile, d'autre part. L'analyse et le contrôle normal par les "pairs" permettront de corriger la plupart de ces situations.

"Une autre option consisterait à s'inspirer, dans l'établissement des normes, des pratiques suivies par les toxicologues depuis de nombreuses décennies : les taux de concentration admissibles des substances toxiques sont fixés en-deçà du niveau où un effet est susceptible de se manifester. L'existence d'un seuil est ainsi toujours postulée mais jamais affurmée. En fait, les toxicologues ont tendance aujourd'hui à se rallier à la conception linéaire, sans seuil, qui prévaut en matière de rayonnements ionisants. Une combinaison judicieuse des deux philosophies (c'esta-dire de toutes celles qui existent) pourrait constituer la solution la plus raisonnable au problème de la protection.

"Je ne propose rien de nouveau, mais je souligne la responsabilité fondamentale des spécialistes de la radioprotection dans l'évaluation critique des problèmes et la présentation des faits au public, présentation conçue de façon à dissiper les craintes et les suspicions.

"Nous ne pouvons quitter le domaine philosophique sans aborder une question qui me préoccupe personnellement depuis le jour où elle a été introduite pour la première fois. Cette question a trait au système en vertu duquel les conditions d'exposition et les doses admissibles varient selon les catégories de personnes.

"Une question surgit logiquement : pourquoi les professionnels devraient-ils être exposés à des doses plus élevées que le reste de la population? La réponse ressort de ce qui précède, mais elle est rarement comprise de cette façon. D'un point de vue philosophique, des arguments solides pourraient être avancés pour justifır la fıxation de normes uniformes pour les professionnels et le public. D'un autre côté, pour des raisons pratiques incontestables et en l'absence de preuves établissant la réalité des dommages subis au moins par les professionnels, l'établissement de normes uniformes entraînerait des coûts appréciables et inacceptables pour un gain qui n'est pas quantifıable." 
Une approche politique a été tentée au sens où " être politique " signifie " être prudent et perspicace dans l'élaboration et la mise en œuvre de mesures d'intérêt général " (définition du dictionnaire). Malheureusement, cette approche a été confondue avec la politique partisane, c'est-à-dire la branche de la politique mettant en cause principalement des actions de type électoraliste.

Aux États-Unis, tous les problèmes concernant les rayonnements ont relevé, de 1946 à 1977, du Joint Committee on Atomic Energy, organisme relevant du Sénat et de la Chambre des représentants. Contestée par les écologistes, cette commission a été remplacée, selon une loi de 1977, par quelques deux douzaines de commissions souvent dépourvues de personnel stable et de pouvoir de contrôle. Leurs responsables ont souvent une connaissance insuffisante des problèmes des rayonnements et entretiennent parfois le trouble du public en appelant à comparaître des personnes connues pour faire des gros titres, mais dont la bonne foi est mise en cause par leurs confrères.

Les media jouent un rôle majeur dans l'éducation du public. Nous devons les persuader qu'ils ont une obligation nationale dans ce domaine. " J'ai souligné en 1977 à Paris (*) que les media avaient failli à leur tâche en insistant sur le côté sensationnel de l'histoire au détriment de faits en réalité inquiétants. Je fus sévèrement critiqué dans le journal Le Monde qui faisait valoir que les journalistes ne font que rapporter les faits. "

Le premier amendement de la Constitution des États-Unis confirme la liberté de la presse, mais comporte aussi une obligation implicite, celle de rapporter intégralement et fidèlement les nouvelles.

"Le principe a été constamment et continuellement méconnu en ce qui concerne les rayonnements ionisants, domaine que je connais assurément bien. La presse rapporte et met en relief les nouvelles et les détails dont elle croit que le public souhaite entendre parler et qui l'aideront à vendre ses journaux, ce qui est apparemment pour elle l'essentiel. Les media doivent faire de l'argent pour vivre. Ils doivent vendre leurs produits et éviter de perdre du temps sur des choses qui ne paient pas. Aussi, consciemment ou non, un processus de sélection s'enclenche. Les journaux disent qu'ils répondent aux vaux du public. Pour ce qui est de ce dernier, il ne peut prendre que ce qu'on lui donne. Malheureusement, nous n'avons pas les moyens d'exercer cette énorme pression économique qui obligerait la presse à contribuer à l'éducation du public dans le domaine des rayonnements et, vraisemblablement dans de nombreux autres domaines également. "

\section{LOIS ET RÈGLEMENTS}

Ce sont des armes à double tranchant; ils sont essentiels pour uniformiser et contrôler l'ensemble des utilisateurs. Mais en même temps, ils tendent à étouffer l'initiative et l'innovation, invitent aux procès et aux actions judiciaires. Je me souviens d'un avocat conseillant à un jeune confrère " allez vers l'Ouest, c'est un domaine d'avenir». Il ajouterait aujourd'hui "tournez-vous vers le droit nucléaire et des rayonnements; c'est un domaine d'avenir.»

(1) Colloque S.F.R.P. sur les implications psycho-sociologiques du développement de l'industrie nucléaire, Paris, janvier 1977.

RADIOPROTECTION 
Les 17 agences et les 24 commissions parlementaires qui s'intéressent aux rayonnements se disputent prestige et budget, en multipliant les doubles emplois. La lutte qui a opposé la N.R.C. $\left({ }^{2}\right)$ et l'E.P.A. $\left({ }^{3}\right)$ pour la fixation des normes, sous le regard anxieux du Département de l'Énergie, est un exemple de ces luttes internes. Le rapport Kemeny sur l'accident de Three Mile Island dénonçait le danger de l'abus des règlements : " Nous sommes convaincus que les règlements ne peuvent à eux seuls assurer la sécurité. En vérité, lorsque la réglementation est aussi volumineuse et complexe qu'elle l'est actuellement, elle peut, du point de vue de la sécurité nucléaire, constituer un facteur négatif. "

Dans cet esprit je tiens à dire que l'application du principe ALAP ou ALARA $\left({ }^{4}\right)$ (aussi bas que possible), principe qui n'est pas scientifiquement ni techniquement prouvé, devrait être limitée pour ne pas décourager l'initiative ou l'innovation. Le concept de " possibilité » ou de " praticabilité » est bon en soi, sauf lorsqu'on veut imposer une "praticabilité " minimale.

"Pour avoir une autorité réglementaire, il est nécessaire d'avoir au préalable une législation sur les rayonnements. L'étape suivante consistera à élaborer un modèle de règlements, ce qui suppose la mise en place d'une équipe émargeant sur les fonds publics. Le problème est à ce stade qu'aucune législation sur les rayonnements ni aucun dispositif réglementaire n'est fixé à jamais. Ils paraissent devoir évoluer; cette évolution débouchera avant peu sur la constitution d'une nouvelle équipe dans quelque bureau, sous la direction d'un chef de service, secondé par un chef adjoint, un auxiliaire et même quelques employés. L'étape suivante verra évidemment la création d'un nouveau bureau.

"Cependant, une fois accomplie la mission qui lui était impartie, le service ou le bureau se retrouvera sans rien à faire. Ce qui, naturellement, ne saurait se produire. Reste donc à trouver de nouvelles missions en remplacement de la précédente. Le principe de base de la bureaucratie est qu'elle est condamnée à se développer ou à dépérir. Elle ne peut même pas rester en l'état, si bien que nous risquons d'être pris à brève échéance dans un réseau de règlements de plus en plus complexes et restrictifs qui déféreront toujours davantage à l'État la responsabilité d'actions qui étaient primitivement destinées à promouvoir l'initiative, l'innovation et l'usage réel des bienfaits de nos sciences et techniques. "

La difficulté est accrue aux États-Unis du fait de l'existence de règlements au niveau fédéral, au niveau des États et parfois au niveau des villes elles-mêmes, d'où le danger de multiplier le dispositif.

"A moins de faire quelque chose pour contrôler notre boulimie de règlements et de lois dans pratiquement tous les domaines dans ce pays, nous nous exposons à de réelles difficultés. J'en suis arrivé à parler des problèmes médico-légaux mais je dois mentionner une phrase courante aux États-Unis et probablement partout ailleurs : "Si vous avez des problèmes, faites une loi. " Ce qui n'est pas dit, c'est que nous voulons pour l'essentiel contribuer à l'instauration d'un paradis juridique - pour autrui.

$\left({ }^{2}\right)$ N.R.C. : Nuclear Regulatory Commission.

$\left({ }^{3}\right)$ E.P.A. : Environmental Protection Agency.

$\left({ }^{4}\right)$ ALAP. : As low as practicable. ALARA : As low as readily achievable. 
"Une autre diffıculté d'ordre juridique concerne la santé. la sécurité et les soins médicaux de la naissance à la mort. On évoquera ici l'indemmisation des travailleurs dans l'industrie nucléaire et des rayonnements. (Je me hâterai d'ajouter que je considère personnellement l'indemnisation des travailleurs comme une obligation légitime à la charge de l'industrie.) Un nombre croissant de litiges nés de radiolésions sont réglés aux États-Unis, au profıt des travailleurs, habituellement par les conseils d'indemnisation mais aussi par les différents tribunaux. Les individus avaient été,dans la plupart des cas exposés,à des doses très faibles mais n'en avaient pas moins développé des tumeurs malignes. Pour autant que j'ai eu connaissance de ces cas, la plupart n'avaient pas été exposés leur vie durant à des doses supérieures à 5 ou 10 rads, lesquelles avaient été étalées sur une période de plusieurs années.

"Nous savons très bien que les rayonnements peuvent provoquer l'apparition de tumeurs malignes encore que, en l'état actuel de nos connaissances touchant la relation dose-effet, leur probabilité soit liée à des taux d'exposition très éloignés de ceux mentionnés. Quelle que soit la procédure choisie, la plupart des plaignants ont obtenu gain de cause, bien qu'il soit établi dans certains cas, que les rayonnements ne sont pas connus pour avoir développé telle ou telle forme particulière de cancer.

"Le problème n'est pas que le jugement rendu en faveur du plaignant soit nécessairement répréhensible mais bien plutôt que notre appareil judiciaire ait créé des précédents. Les tribunaux ont réglé en droit des questions scientifıques qui ne pouvaient être résolues par la science. Ils ont " établi " par voie de jurisprudence une relation dose-effet qui n'est pas corroborée à l'heure actuelle par les observations scientifiques.

"Les procès ont été, dans certains cas, extrêmement coûteux et les décisions injustifiables et déraisonnables. Prenons, par exemple, l'affaire Silkwood $\left({ }^{5}\right)$. J'ai déclaré en diverses occasions, parfois sérieusement, parfois en matière de plaisanterie, qu'il serait moins coûteux et peut-être plus humain de décider que toute personne qui souffrirait de cancer après avoir travaillé sous rayonnements à un moment quelconque de son existence aurait droit à des soins gratuits et que sa famille devrait bénéficier d'une indemnité normale. Compte tenu des programmes nationaux de santé en vigueur ou en projet, pareille prise en charge ne représenterait qu'une goutte dans l'océan. »

\section{ÉCONOMIE}

Toute mesure de protection repose sur une évaluation et une planification qui prend en compte les considérations scientifiques, la production, et les moyens techniques. Le problème est d'évaluer les risques, le coût d'une plus grande sécurité et les gains à en attendre. On dit souvent à ce propos qu'on soupèse les risques et les profits. Ce n'est pas facile dans le domaine médical; c'est encore plus difficile dans le cas des réacteurs nucléaires, tant les risques sont déjà

( $\left.^{5}\right)$ Karen Silkwood, employée chez Kerr McGee, trouvée morte dans sa voiture. On a relevé sur elle des traces de plutonium dont l'enquête n'a jamais permis d'identifier l'origine. 
faibles au départ. Nous devons diviser par 2 ou par 5 ce que nous ne connaissons pas au départ. On a vu une agence fédérale démontrer qu'une limitation du taux moyen d'irradiation peut engendrer une économie pour le contribuable en réduisant les cas d'hospitalisation et de soins médicaux pour des effets imaginaires. Une personne étrangère à l'agence, et sur des bases identiques, aboutissait à la conclusion inverse.

"Je crains qu'en matière de rayonnements en général et de radioprotection en particulier, toute tentative d'évaluation ne reste très subjective et basée sur des opinions individuelles de sorte qu'elle se révèle incommode dans la plupart des cas. Il y a de nombreux facteurs des deux côtés de la question tels que ceux relevés plus haut où le spécialiste de la radioprotection peut et doit jouer un rôle intelligent et fécond, tout en évitant les partis pris et les aspects partisans de la situation.

\section{Mesures Correctives POSSIBLes}

Je voudrais d'abord améliorer la communication à l'intérieur des groupes scientifiques et auprès du public et, pour cela, développer le respect et la confiance mutuelle. Le scientifique a joui dans le passé d'un haut degré de respect et de confiance; il ne suscite aujourd'hui que méfrance qu'il faut connaître et corriger.

"Les rayonnements conservent pour le public une aura de mystère, presque de mysticisme, et, pour des raisons dont certaines étaient valables et d'autres non, le public en est venu à réaliser qu'en diverses occasions, il a été abusé en matière de rayonnements par les agences gouvernementales ou qu'il ne lui a pas été donné de connaître toute la vérité. Quant aux responsabilités de l'État dans ces affaires, on peut probablement faire valoir qu'à l'époque où l'information faisait l'objet de rétentions ou de distorsions, il y avait des raisons économiques ou politiques considérées comme valables pour le justifıer. En une période de menaces internationales, aventures en Asie, subversions dans le pays, problèmes en Iran, la désinformation du public peut se défendre. Je n'aperçois pas à l'heure présente de raisons de nature à justifıer l'actuelle dissimulation mais, en même temps, il est pratiquement impossible de revenir en arrière et de corriger la plupart des erreurs antérieures. "

Nous devons détecter les nouvelles erreurs, les expliquer et les corriger, ce qui représente de grands efforts pour le monde scientifique mais qui permettra d'identifier et d'isoler "la petite poignée de prophètes de malheur qui fournissent aux media et au public des informations délibérément mensongères et généralement partiales en matière de rayonnements"..

Ce problème de l'information a été l'un des thèmes majeurs du colloque organisé à Paris en 1977 par la Société française de Radioprotection (S.F.R.P.) sous le titre Colloque sur les conséquences psycho-sociologiques du développement de l'industrie nucléaire. Il est ressorti des débats que l'inquiétude, la méfiance et l'incompréhension du public à l'égard du problème nucléaire dans son ensemble trouvaient, pour l'essentiel, sa source dans une éducation inadéquate (M.Tubiana). 
"Je pense que nos media, journaux, revues, radio et télévision portent unc lourde responsabilité dans les peurs et les appréhensions que connaît le public ¿i l'égard des rayonnements...

"Où les gens puissent-ils les informations? Principalement dans la pressc. et j'estime que la presse a failli à ses obligations non écrites qui découlent des libericís

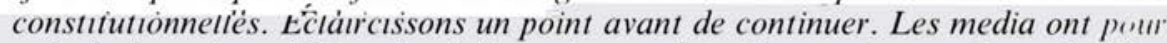
principale raison d'être de réaliser des profıts dans notre système de lilurc entreprise. Ils bénéfıcient en même temps d'une protection particulière en vertu cilı premier amendement dans la collecte et la diffusion des informations sans parlir des éditoriaux qui rapportent des opinions, non des informations. Nous ne devons prendre aucune mesure qui serait susceptible de porter atteinte à cette liberti: fondamentale. Mais peut-être devrions-nous envisager certaines restrictions oti sanctions pour manquement aux obligations. Exigeons,par exemple,des media qus. dans la relation d'un événement, ils rapportent tout ce qui s'est produit et non pa. seulement les faits qui entrent dans la ligne de la publication. Des erreur: d'observation sont possibles, elles sont excusables. Sont, en revanche, totalement inacceptables les erreurs commises en retenant certains faits à l'exclusion d'un certain nombre d'autres. "

Les directeurs de journaux reconnaissent souvent eux-mêmes qu'ils ne s'intéressent à la matière imprimée que dans la mesure où elle leur paraît de nature à accroître la vente de leur marchandise. La seule alternative à cette situation serait des media contrôlés et financés par l'État, qui refléteraient totalement la politique du gouvernement.

"Ce serait intolérable. Il nous reste à nous faire à l'idée qu'une presse indépendante ne peut survivre qu'en faisant des bénéfıces. Qu'est-il arrivé dans les pays où l'on a cru pouvoir disposer d'une liberté totale sans responsabilité."

"En ce qui concerne les rayonnements, une expérience intéressante, noble et peut-être critique, consisterait pour la presse à s'astreindre pendant plusieurs années à ne rapporter clairement, exactement et correctement, que l'information qui aura été préalablement vérifiée. Elle devra dans le même temps s'abstenir de faire état de l'opinion du journal, de deviner les sens cachés, de donner des interprétations techniques pour lesquelles les journalistes, si bien formés soient-ils, et leurs directeurs ne sont pas qualifiés. Si les media limitaient là leurs ambitions, ils devraient s'assurer l'aide de spécialistes de la radioprotection, formés et responsables, et je suis certain qu'elle leur serait assurée. Si grande soit l'intégrité des spécialistes mis à contribution, il n'en faudrait pas moins un contrôle technique pour s'assurer de leur objectivité et de leur impartialité. Comme je l'ai dit, ce serait une expérience noble. Je défie les organes d'information responsables de la tenter pendant peut-être plusieurs années. Si cette expérience s'avérait concluante sous une forme ou une autre, tous les media s'y rallieraient et, pour ce qui concerne les rayonnements, nous nous trouverions, de nouveau, bien informés des problèmes. "

Un autre reproche doit être fait aux media; c'est l'usage de quelques personnes manifestement déphasées par rapport à la communauté scientifique. Aux États-Unis qui comptent quelques 6000 spécialistes de la santé et radiologues, les media font appel à une demi-douzaine de personnes (je les appellerai les six Américains) qui diffusent des informations délibérément mensongères concernant les rayonnements. 
Ils forment un groupe recherché par la presse à sensation; n'ayant plus rien à perdre vis-à-vis de la communauté scientifique qui les a rejetés, ils peuvent transgresser la vérité sans courir le même risque qu'un interlocuteur honnête qui doit intervenir avec une rigueur scientifique totale.

On observe le même phénomène avec certains ouvrages populaires, souvent criblés de demi-vérités, de contre-vérités, et révélateurs d'une ignorance fondamentale du sujet. Leurs auteurs pourraient écrire avec la même autorité et le même profit sur la soupe de légumes ou le tourisme en Inde. Le public se laisse malheureusement abuser par ces erreurs et ces tromperies, d'autant plus qu'elles se retrouvent dans presque tous les articles, ce qui accroît leur degré de crédibilité.

Le succès commercial n'est pas étranger à ce désir d'écrire, même chez de bons scientifiques comme Gofmann et Tamplin qui ont profité de leur réputation pour critiquer la politique nucléaire et réclamé l'abaissement des normes.

Enfın, le lien avec la bombe atomique est une pratique insidieuse couramment utilisée. Ce phénomène est apparu après l'explosion des bombes expérimentales à forte puissance dans les années cinquante. Tout document ou tout reportage sur un incident même mineur débute par une évocation de la bombe, d'une explosion, de retombées, etc. Cela s'est produit à Three Mile Island où la présence de la bulle d'hydrogène a été décrite en termes de bombe. Or, on savait que tout rentrerait dans l'ordre, qu'il n'y aurait pas de « fusion " ni de cratère jusqu'en Chine.

"Le Washington Post a reconnu lui-même: La presse, quelle que soit sa nationalité, vit de viande rouge, à savoir des désastres, des tragédies, des conflits, guerres, assassinats, ou massacre de Johnestown, coup de grisou dans les mines.

"L'accident de Three Mile Island était de la viande rouge, d'une espèce jamais expérimentée par la presse; toute la belle fıbre d'un Delmonico. C'était l'histoire d'une perte de contrôle technique, de l'homme contraint d'abandonner son foyer sous la menace de l'atome pacifique, la perspective d'une partie du littoral oriental transformé en désert irradié. "

\section{CRÉDIBILITÉ DES SCIENTIFIQUES}

Elle doit être restaurée. Les scientifiques sont des êtres humains, des citoyens, avec leurs problèmes de santé, de familles comme les autres. Ils sont également exposés à des erreurs de jugement, comme tout un chacun.

Ils devraient être appréciés par le fait qu'ils ne se sont sûrement pas engagés dans la recherche pour des raisons financières. Leur récompense première est la réputation, l'objectivité, l'honnêteté intellectuelle. Dans tous les cas, ils ne démarrent jamais avec une idée préconçue de ce que sera le résultat final. A moins d'accepter de perdre sa réputation, aucun scientifique ne peut être suborné par les personnes ou organismes qui financent ses recherches. Il serait ridicule de supposer que le corps tout entier des scientifiques engagés dans la recherche nucléaire trahirait ses normes professionnelles. 


\section{CONCLUSION}

Il faut mettre un terme à cette succession apparemment sans fin de commissions et d'auditions sur le problème des faibles doses de rayonnements ionisants. La réponse finale et complète à la relation dose-effet pour de faibles niveaux (5 à 10 rads) et à faible débit n'est pas en vue; mais nous savons que les effets sont minimes pour autant qu'ils existent et ils ne peuvent être identifiés en tant que tels parmi tous les autres effets similaires.

La porte a été ouverte à une spéculation effrénée, tout le monde, scientifiques, commissions et agences gouvernementales, media ayant laissé le monstre sortir de sa boîte. Personne n'est sans reproche sur ce point. Tous doivent apprendre à travailler main dans la main pour informer correctement le public, en respectant les principes de liberté et les obligations qu'elle implique.

\section{Lauriston S. TAYLOR}

Docteur ès-sciences de l'Université de Pennsylvanie.

1922, Bell Telephone Laboratories.

1927, National Bureau of Standards (Chef de la Division « Atomics and radiations Physics »). Premières normes sur la dosimétrie des rayons $\mathrm{X}$.

1948, Détaché à l'Atomic Energy Commission (A.E.C.), responsable de la " Biophysics Branch ".

1962, Directeur au National Bureau of Standards.

1965, Conseiller du Président de l'Office of Emergency Preparedness.

Président honoraire du N.C.P.R. (National Council on Radiation Protection).

Président honoraire de l'I.C.R.U. (Commission internationale pour les unités et mesures radiologiques).

Membre de la C.I.P.R. 


\section{LE CENTRE INTERNATIONAL DE RADIOPATHOLOGIE}

Étant donné le développement des utilisations des rayonnements ionisants et l'accroissement corrélatif des affections radiopathologiques, l'Organisation mondiale de la Santé (O.M.S.) a décidé de créer trois Centres de collaboration en radiopathologie. Ces Centres doivent remplir trois missions : d'intervention médicale, d'assistance sanitaire, de recherche scientifıque.

L'intervention médicale consiste à accueillir en vue de leur diagnostic et de leur traitement des personnes victimes de brûlures radiologiques ou d'intoxications radioactives.

L'assistance sanitaire a pour but d'aider les pays membres de l'O.M.S. à résoudre les problèmes posés par d'éventuels accidents nucléaires, en particulier l'organisation des plans d'urgence médicaux.

La recherche scientifıque porte sur les études de radiopathologie clinique et épidémiologique.

Les Centres agissent dans le cadre des Bureaux régionaux de l'O.M.S. Un centre est implanté à Oak Ridge aux U.S.A., pour l'Amérique du Nord et du Sud; un autre à Paris, en France, pour l'Europe (orientale comprise), l'Afrique et le Proche Orient; un Centre est envisagé au Japon pour l'Asie orientale et méridionale.

En ce qui concerne le Centre parisien, l'O.M.S. a demandé qu'il comprenne les éléments compétents en radiopathologie de l'Institut Curie et du Département de Protection du Commissariat à l'Énergie atomique (C.E.A.). En effet, la diversité des missions impose que tous les services et laboratoires capables d'apporter leur expérience participent aux activités du Centre.

Le Service de radiopathologie de l'Institut Curie devient l'élément principal en matière d'intervention médicale. Les Services de protection sanitaire du C.E.A. apportent leur compétence en assistance sanitaire. Les deux participent aux recherches cliniques et épidémiologiques en radiopathologie. En vue d'obtenir une bonne cohésion de l'ensemble, un protocole d'accord est établi entre l'Institut Curie et le Commissariat à l'Énergie atomique. Il précise les modalités de fonctionnement du Centre international de radiopathologie agrée par l'O.M.S. à l'Institut Curie. Il est placé sous la direction du chef du Service de radiopathologie, le docteur Henri JAMMET.

C'est un honneur pour l'Institut Curie d'avoir été choisi par l'O.M.S. pour agir en tant que Centre de collaboration en radiopathologie. Ceci est dû au fait que l'Institut Curie a créé, il y a plus de 20 ans, le premier service de radiopathologie au monde, suite au souhait exprimé avant sa mort par $\mathrm{M}^{\mathrm{me}}$ Irène Joliot-CuRIE. L'O.M.S. reconnaît ainsi la réputation de l'Institut Curie où ont été traités de nombreux malades français et étrangers, notamment les chercheurs yougoslaves en 1958 et les victimes algériennes en 1978. 